\title{
Adherence to Alternative Healthy Eating Index in relation to depression and anxiety in Iranian adults
}

\author{
Parvane Saneei ${ }^{1,2,3}$, Maryam Hajishafiee ${ }^{1,2,3}$, Ammar Hassanzadeh Keshteli ${ }^{4,5}$, Hamid Afshar ${ }^{6}$, \\ Ahmad Esmaillzadeh ${ }^{7,8_{*}}$ and Peyman Adibi $^{5}$ \\ ${ }^{1}$ Food Security Research Center, Isfahan University of Medical Sciences, PO Box 81745-151, Isfahan, Iran \\ ${ }^{2}$ Students' Research Committee, Isfahan University of Medical Sciences, PO Box 81745-151, Isfahan, Iran \\ ${ }^{3}$ Department of Community Nutrition, School of Nutrition and Food Science, Isfahan University of Medical Sciences, \\ PO Box 81745-151, Isfahan, Iran \\ ${ }^{4}$ Department of Medicine, University of Alberta, PO Box 7-142, Edmonton, Alberta, Canada \\ ${ }^{5}$ Integrative Functional Gastroenterology Research Center, Isfahan University of Medical Sciences, PO Box 81745-151, \\ Isfahan, Iran \\ ${ }^{6}$ Psychosomatic Research Center, Department of Psychiatry, Isfahan University of Medical Sciences, PO Box 81745-151, \\ Isfahan, Iran \\ ${ }^{7}$ Department of Community Nutrition, School of Nutritional Sciences and Dietetics, Tehran University of Medical Sciences, \\ PO Box 14155/6117, Tehran, Iran \\ ${ }^{8}$ Diabetes Research Center, Endocrinology and Metabolism Clinical Sciences Institute, Tehran University of Medical Sciences, \\ PO Box 14155/6117, Tehran, Iran
}

(Submitted 19 November 2015 - Final revision received 7 March 2016 - Accepted 11 April 2016 - First published online 18 May 2016)

\section{Abstract}

Earlier studies have shown a protective association between adherence to healthy eating guidelines and mental disorders in Western nations; however, data in this regard are limited from the understudied region of Middle East. We examined the association between adherence to healthy eating guidelines, as measured by Alternative Healthy Eating Index (AHEI)-2010, and prevalence of anxiety and depression in a large sample of Iranian adults. In this cross-sectional study, data on dietary intakes of 3363 adult participants were collected using a validated dish-based 106-item semi-quantitative FFQ. Adherence to healthy eating was quantified using AHEI-2010, as suggested by earlier publications. The Iranian validated version of Hospital Anxiety and Depression Scale was used to assess anxiety and depression in study participants. Data on other covariates were gathered using a pre-tested questionnaire. Overall, the prevalence of anxiety and depression was $15 \cdot 2 \%(\mathrm{males}$ $10.8 \%$ and females $18.3 \%$ ) and $30.0 \%$ (males $22.9 \%$ and females $35.1 \%$ ), respectively. After controlling for potential confounders, those in the top quartile of AHEI-2010 had a $49 \%$ lower chance of anxiety (OR 0.51; $95 \%$ CI 0.35, 0.72) and a $45 \%$ lower odds of depression (OR 0.55; $95 \%$ CI $0.42,0.72$ ), compared with those in the bottom quartile. Stratified analysis by sex revealed that women in the highest categories of AHEI-2010 had a 49\% lower odds of having anxiety and depression, after adjustment for confounders, but no significant association was found in men. In addition, among individuals who were 40 years old or younger, those with high adherence to AHEI-2010 were 58 and $51 \%$ less likely to have anxiety and depression, compared with those with less adherence. Adherence to healthy eating was inversely associated with a lower chance of anxiety and depression in Iranian adults. Prospective studies are required to confirm these associations in Middle-Eastern populations.

Key words: Alternative Healthy Eating Index: Diet quality: Depression: Anxiety

Common mental disorders, major depression and anxiety in particular, are highly prevalent in both developed and developing countries ${ }^{(1,2)}$. It has been estimated that on average one in five adults, particularly women, suffer from common mental disorders in their lifetime ${ }^{(2)}$. Reports from Iran have revealed that anxiety and depressive symptoms affect about $21 \cdot 0$ and $20 \cdot 8 \%$ of the adult population, respectively ${ }^{(3)}$.
A large body of evidence has indicated the association of modifiable risk factors including poor diet quality and physical inactivity with common mental disorders ${ }^{(4,5)}$. Observational studies have indicated that dietary intakes of single nutrients, foods and food groups including $n-3$ long-chain fatty acids, fruits, vegetables, whole grains and fish are associated with a lower risk of depression ${ }^{(6,7)}$. However, diet-disease relations

\footnotetext{
Abbreviation: AHEI, Alternative Healthy Eating Index.
}

* Corresponding author: A. Esmaillzadeh, fax +98 313668 1378, email esmaillzadeh@nutr.mui.ac.ir 
have mostly been recommended to be examined through dietary pattern approach rather than individual foods and nutrients ${ }^{(5,8)}$. Prospective studies have suggested that adherence to healthy dietary pattern, as measured by Alternative Healthy Eating Index (AHEI), was associated with a reduced risk of recurrent depressive symptoms in women, but not in men ${ }^{(9)}$. This favourable association has been attributed to components of AHEI including vegetables, fruits and the ratio of PUFA:SFA ${ }^{(9)}$. Moreover, it has been reported that AfricanAmerican, as well as Caucasian, adults living in Baltimore who had high diet quality, as measured by Healthy Eating Index (HEI)-2005, had fewer depression symptoms ${ }^{(10)}$. Similar findings have also been reported in National Health and Nutrition Examination Survey study, in which higher HEI was associated with a lower risk of depression in the adult population ${ }^{(11,12)}$. Findings from a meta-analysis of observational studies have also revealed a significant inverse association between healthy dietary patterns, identified by factor analysis, and risk of depression ${ }^{(8)}$.

Although earlier studies have reported a protective association between healthy eating pattern and mental disorders in Western nations, data in this regard are limited in the understudied region of the Middle East, where almost all lifestyle components are significantly different from those in Western countries. In particular, dietary intakes of Middle-Eastern population have their own characteristics: large intake of carbohydrates mostly in the form of refined grains, high intakes of trans and SFA and low consumption of fruits and vegetables along with lack of alcohol intake makes their dietary pattern of great interest for the assessment of diet-disease relations. In addition, different from Western people, stressful life is highly prevalent in this region, which might further complicate diet-disease relations in this area. Therefore, we aimed to examine the association of AHEI with anxiety and depression in a large sample of Iranian population.

\section{Methods}

\section{Participants}

This cross-sectional study was carried out within the framework of the Study on the Epidemiology of Psychological-Alimentary Health and Nutrition ${ }^{(13)}$, a project that was performed on Iranian general adults working in fifty different healthcare centres affiliated to Isfahan University of Medical Sciences (IUMS). The project included two main phases. In the first phase, a detailed self-administered questionnaire on sociodemographic factors and dietary behaviours was distributed among 10087 apparently healthy adults, and 8691 individuals returned the completed questionnaires (response rate: 86.16\%). No significant difference was found between the demographic data of those who returned the completed questionnaires and those who did not. In the second phase, another set of questionnaires was sent out to the same participants to obtain data on psychological distress and mental disorders (response rate: $64.64 \%$ ). After merging data from these two phases, we had complete information for 4633 participants. Data for 1606 participants could not be used in the merging process because (1) some of them had no information at phase 1 (did not complete the questionnaires of first phase), and (2) some did not complete their identification code in phase 1 or phase 2 . In the current study, participants with energy intakes outside the range of $3347-17573 \mathrm{~kJ} / \mathrm{d}(800-4200 \mathrm{kcal} / \mathrm{d})$, as under-reporters and over-reporters of energy intake, were excluded $(<3347 \mathrm{~kJ} / \mathrm{d}$ (<800 kcal/d), $n$ 128; > $17579 \mathrm{~kJ} / \mathrm{d}$ (>4200 kcal/d), $n$ 488). We also excluded individuals with missing data on dietary intakes, outcome and covariate variables ( $n$ 654). These exclusions resulted in a data set of 3363 adults who had complete information on dietary intakes and mental health. A comparison of the general characteristics of those who were included in the current study with those who were excluded revealed that there was no significant difference. All participants provided signed informed written consent. The study was ethically approved by the Medical Research Ethics Committee of IUMS, Isfahan, Iran.

\section{Assessment of dietary intakes}

Data on dietary intakes were collected using a Willett-format dish-based 106-item semi-quantitative FFQ, which was designed and validated specifically for Iranian adults ${ }^{(14)}$. Detailed information about the design, foods included and the validity of this questionnaire has been reported elsewhere ${ }^{(14)}$. In brief, the FFQ contained information on the frequency of consumption of foods or dishes over the past year, along with portion sizes commonly used in Iran. A daily value for each item was calculated based on food composition, specified portion size and the average reported frequency. Nutrient intakes were calculated by summing up the nutrient contents of all foods and dishes. Nutritionist IV software, which was modified for Iranian foods, was used to obtain nutrient intakes of each participant. Overall, our previous investigations revealed that the FFQ provides reasonably valid and reliable measures of the average long-term intakes of foods ${ }^{(15)}$, food groups $^{(16)}$ and nutrients ${ }^{(17)}$.

\section{Assessment of adherence to Alternative Healthy Eating Index}

To examine the adherence to the healthy eating guidelines, we used AHEI-2010 as previously described ${ }^{(18)}$. AHEI-2010 consisted of eleven components: fruit, vegetables, whole grains, nuts and legumes, long-chain $n-3$ fats (DHA and EPA), PUFA, alcohol consumption, sugar-sweetened drinks and fruit juice, red and processed meats, trans-fat and $\mathrm{Na}$. In the current study, alcohol consumption was not included into the score, because of the lack of information in the original data set. To construct the index, first we obtained energy-adjusted intakes of the above-mentioned components by using the residual method ${ }^{(19)}$. Next, participants were classified based on decile categories of energy-adjusted intakes of these components. As scoring by deciles would be least prone to misclassification, we used decile categories of components instead of quantitative classifications. Individuals in the highest deciles of fruits, vegetables, whole grains, nuts and legumes, long-chain $n$ - 3 fats and PUFA were given a score of 10 , and those in the lowest deciles of these items were given a score of 1 . Individuals in the other deciles of these components were assigned the corresponding scores. Regarding sugar-sweetened drinks and fruit juice, red and processed meat, trans-fatty acids and $\mathrm{Na}$ 
intake, the lowest deciles were given a score of 10 and the highest deciles were given a score of 1 . Those in deciles 9, 8, 7, $6,5,4,3$ and 2 of these components were given scores of 2, 3, 4, 5, 6, 7, 8 and 9, respectively. The whole AHEI-2010 was computed through summing up the scores of its components ranging from 10 to 100 .

\section{Assessment of outcomes}

The Iranian validated version of Hospital Anxiety and Depression Scale (HADS) was used to screen for anxiety and depression ${ }^{(20)}$. HADS is a brief and useful questionnaire to assess psychological disorders and to measure the symptoms and severity of anxiety disorders and depression. It contains fourteen items and consists of two subscales: anxiety and depression. Each item includes a four-point scale; higher scores indicate an elevated level of anxiety and depression. Maximum score is 21 for anxiety and depression. Scores of 8 or more on either subscale were considered as psychological disorders, and scores of 0-7 were defined as 'normal' in the current study ${ }^{(20)}$.

\section{Assessment of covariates}

Data on weight $(\mathrm{kg})$ and height $(\mathrm{cm})$ were gathered using a self-reported questionnaire. BMI was calculated as weight in kilograms divided by the square of height in metres. In our validation study on 200 participants from the same population, we found that the correlation coefficient between self-reported and technician-measured weight and height were 0.95 $(P<0.001)$ and $0.83(P<0.001)$, respectively. The correlation coefficient for computed BMI from self-reported values and the one from measured values was $0 \cdot 70(P<0.001)$. These findings indicate that the self-reported values of anthropometric indices provide reasonably valid measures in this population.

Physical activity of study participants was assessed by using a General Practice Physical Activity Questionnaire (GPPAQ). This questionnaire is a simple validated screening tool for ranking adult people's physical activity by focusing on current general activities ${ }^{(21)}$. Participants were classified into four categories: active (>3 h/week), moderately active (1-3h/week), moderately inactive $(<1 \mathrm{~h} /$ week) and inactive (no physical activity), according to the type and intensity of their physical activity in work hours and during the weekends. The validity of the GPPAQ for assessment of habitual physical activity levels has been previously examined ${ }^{(21)}$.

Additional covariate information regarding age, sex, marital status, smoking, education levels, family size, house possession, disease history, current use of anti-psychotic medications (including nortriptyline, amitriptyline or imipramine, fluoxetine, citalopram, fluvoxamine and sertraline) and dietary supplements (including intake of $\mathrm{Fe}, \mathrm{Ca}$, vitamins and other dietary supplements) was obtained using self-administered questionnaires.

\section{Statistical analysis}

Subjects were categorised based on quartiles of AHEI-2010. To compare general characteristics and dietary intakes across quartiles of AHEI-2010, we used one-way ANOVA and $\chi^{2}$ tests where appropriate. We computed age-, sex- and energy-adjusted intakes of nutrients and food groups using ANCOVA. Comparison of dietary intakes across quartiles of AHEI-2010 was performed using ANCOVA with Bonferroni correction. To find the relation between AHEI-2010 and odds of anxiety and depression, we used multivariate logistic regression in different models. First, we controlled for age (years), sex (male/female), energy intake $(\mathrm{kJ} / \mathrm{d}(\mathrm{kcal} / \mathrm{d}))$ and BMI $\left(\mathrm{kg} / \mathrm{m}^{2}\right)$. Additional adjustment was carried out for physical activity (never, <1, 1-3, >3h/week), smoking (smokers/non-smokers/ex-smokers), marital status (single/married), educational level ( $>$ diploma/ $\leq$ diploma), family size ( $>4 / \leq 4$ members), house possession (yes/no), self-reported diabetes (yes/no), current use of anti-psychotic medications (yes/no) and dietary supplements (yes/no) in the second model. Stratified analyses were done by sex (men $v$. women) and age ( $\leq 40 v .>40$ years). To calculate the trend of $\mathrm{OR}$ across increasing quartiles of AHEI-2010, we considered AHEI-2010 quartiles as an ordinal variable. In all analyses, those in the first quartile of AHEI-2010 were considered as the reference category. Two-tailed $P$ values $<0.05$ were considered to be statistically significant. Statistical package for the social sciences software, version 18 was used for all analyses.

\section{Results}

The study sample consisted of 3363 subjects with a mean age of 36.29 (sD 7.87) years; $58.3 \%$ of participants were women. Main characteristics of study participants across quartiles of AHEI-2010 are summarised in Table 1. Individuals in the highest quartile of AHEI-2010 were more likely to be women, older and more educated, compared with those in the lowest quartile. There were no significant differences in other demographic characteristics of participants across categories of AHEI-2010.

Multivariable-adjusted intakes of selected nutrients and food groups across quartiles of AHEI-2010 are provided in Table 2. Greater adherence to AHEI-2010 was associated with higher consumption of protein $(P=0.03)$, carbohydrate, fibre, $n-3$ fatty acids, vitamin $\mathrm{B}_{1}$ and vitamin $\mathrm{B}_{6}$ and lower intakes of energy $(P<0 \cdot 001)$. Participants in the highest quartile of AHEI-2010 had significantly higher intakes of whole grains, fruits and vegetables, low-fat dairy products, nuts and legumes and lower intakes of red meat, refined grains, trans-fatty acids, sugar-sweetened beverages and hydrogenated vegetable oils compared with those in the lowest quartile $(P<0.001$ for all).

The prevalence of anxiety and depression was $15 \cdot 2 \%$ (males $10.8 \%$ and females $18.3 \%$ ) and $30.0 \%$ (males $22.9 \%$ and females $35.1 \%$ ), respectively. The prevalence of anxiety and depression across quartiles of AHEI-2010 is shown in Fig. 1. Greater adherence to AHEI-2010 was associated with a lower prevalence of anxiety. Moreover, depression was significantly less prevalent among individuals in the top quartile of AHEI-2010 compared with those in the bottom quartile.

Multivariable-adjusted OR for anxiety and depression across quartiles of AHEI-2010 in the whole population, as well as stratified by sex and age group, are presented in Table 3. Those in the top quartile of AHEI-2010 had $41 \%$ lower chance of anxiety compared with those in the bottom quartile (OR 0.59; 
Table 1. General characteristics of study participants across quartiles (Q) of Alternative Healthy Eating Index-2010 (AHEI-2010) (Mean values and standard deviations)

\begin{tabular}{|c|c|c|c|c|c|c|c|c|c|}
\hline & \multicolumn{8}{|c|}{ Quartiles of AHEI-2010 } & \multirow[b]{3}{*}{$P^{\star}$} \\
\hline & \multicolumn{2}{|c|}{$Q_{1}$} & \multicolumn{2}{|c|}{$\mathrm{Q}_{2}$} & \multicolumn{2}{|c|}{$\mathrm{Q}_{3}$} & \multicolumn{2}{|c|}{$\mathrm{Q}_{4}$} & \\
\hline & Mean & SD & Mean & SD & Mean & SD & Mean & SD & \\
\hline Age (years) & $35 \cdot 27$ & $7 \cdot 67$ & 35.79 & $7 \cdot 72$ & $36 \cdot 43$ & $7 \cdot 71$ & 37.65 & $8 \cdot 18$ & $<0.001$ \\
\hline Weight (kg) & $69 \cdot 32$ & 13.91 & 68.79 & $13 \cdot 61$ & $68 \cdot 30$ & $12 \cdot 92$ & $68 \cdot 22$ & $12 \cdot 27$ & 0.30 \\
\hline BMI $\left(\mathrm{kg} / \mathrm{m}^{2}\right)$ & 24.65 & 4.43 & $25 \cdot 17$ & 5.43 & $25 \cdot 03$ & 4.25 & $25 \cdot 21$ & 3.59 & 0.05 \\
\hline Female (\%) & \multicolumn{2}{|c|}{$49 \cdot 3$} & \multicolumn{2}{|c|}{$56 \cdot 0$} & \multicolumn{2}{|c|}{61.4} & \multicolumn{2}{|c|}{66.0} & $<0.001$ \\
\hline Married (\%) & \multicolumn{2}{|c|}{$82 \cdot 0$} & \multicolumn{2}{|c|}{$82 \cdot 0$} & \multicolumn{2}{|c|}{$81 \cdot 6$} & \multicolumn{2}{|c|}{$81 \cdot 1$} & 0.91 \\
\hline Education (\%) (>diploma) & \multicolumn{2}{|c|}{$59 \cdot 2$} & \multicolumn{2}{|c|}{$57 \cdot 9$} & \multicolumn{2}{|c|}{$59 \cdot 4$} & \multicolumn{2}{|c|}{67.9} & 0.01 \\
\hline Family size $(\%)(>4)$ & \multicolumn{2}{|c|}{$14 \cdot 3$} & \multicolumn{2}{|c|}{$12 \cdot 6$} & \multicolumn{2}{|c|}{$12 \cdot 8$} & \multicolumn{2}{|c|}{11.3} & 0.38 \\
\hline House possession (\%) & \multicolumn{2}{|c|}{$57 \cdot 8$} & \multicolumn{2}{|c|}{$55 \cdot 1$} & \multicolumn{2}{|c|}{$58 \cdot 7$} & \multicolumn{2}{|c|}{$61 \cdot 6$} & 0.04 \\
\hline Diabetes (\%) & \multirow{2}{*}{\multicolumn{2}{|c|}{$\begin{array}{l}1.3 \\
4.8\end{array}$}} & \multirow{2}{*}{\multicolumn{2}{|c|}{$\begin{array}{l}1.8 \\
4.9\end{array}$}} & \multicolumn{2}{|c|}{1.7} & \multicolumn{2}{|c|}{$2 \cdot 4$} & 0.40 \\
\hline Anti-psychotic medications (\%)† & & & & & \multicolumn{2}{|c|}{$7 \cdot 2$} & \multicolumn{2}{|c|}{$5 \cdot 1$} & 0.07 \\
\hline Dietary supplement use (\%)‡ & \multicolumn{2}{|c|}{$27 \cdot 5$} & \multicolumn{2}{|c|}{$28 \cdot 4$} & & & & & 0.09 \\
\hline Smokers (\%) & \multicolumn{2}{|c|}{$4 \cdot 6$} & \multicolumn{2}{|c|}{$2 \cdot 8$} & & & & & 0.07 \\
\hline Physically active (\%) ( $\geq 1 \mathrm{~h} /$ week) & & & & & & & & & 0.79 \\
\hline Obese (\%)§ & & & & & & & & & 0.34 \\
\hline
\end{tabular}

* Obtained from ANOVA for continuous variables and $x^{2}$ test for categorical variables.

† Anti-psychotic medications included the intake of nortriptyline, amitriptyline or imipramine, fluoxetine, citalopram, fluvoxamine and sertraline.

‡ Dietary supplements included the intake of $\mathrm{Fe}, \mathrm{Ca}$, vitamins and other dietary supplements.

$\S \mathrm{BMI} \geq 30 \mathrm{~kg} / \mathrm{m}^{2}$.

Table 2. Multivariable-adjusted intakes of selected nutrients and food groups of study participants across quartiles (Q) of Alternative Healthy Eating Index-2010 (AHEl-2010)*

(Mean values and standard deviations)

\begin{tabular}{|c|c|c|c|c|c|c|c|c|c|}
\hline & \multicolumn{8}{|c|}{ Quartiles of $\mathrm{AHEI}-2010$} & \multirow[b]{3}{*}{$P \dagger$} \\
\hline & \multicolumn{2}{|c|}{$Q_{1}$} & \multicolumn{2}{|c|}{$\mathrm{Q}_{2}$} & \multicolumn{2}{|c|}{$\mathrm{Q}_{3}$} & \multicolumn{2}{|c|}{$\mathrm{Q}_{4}$} & \\
\hline & Mean & SD & Mean & SD & Mean & SD & Mean & SD & \\
\hline Energy (kJ/d) & 10794.89 & $3392 \cdot 64$ & 9758.43 & 3368.62 & 9219.57 & 3367.79 & $10219 \cdot 13$ & 3394.06 & \\
\hline Energy (kcal/d) & $2580 \cdot 04$ & $810 \cdot 86$ & $2332 \cdot 32$ & $805 \cdot 12$ & 2203.53 & 804.92 & 2442.43 & $811 \cdot 20$ & $<0.001$ \\
\hline \multicolumn{10}{|l|}{ Nutrients } \\
\hline Proteins (\% of energy) & $14 \cdot 62$ & $2 \cdot 13$ & 14.83 & $2 \cdot 15$ & 14.88 & $2 \cdot 30$ & 14.96 & $2 \cdot 14$ & 0.03 \\
\hline Fats (\% of energy) & $36 \cdot 68$ & $6 \cdot 39$ & 38.41 & $6 \cdot 20$ & $37 \cdot 68$ & $6 \cdot 32$ & $37 \cdot 16$ & $6 \cdot 44$ & $<0.001$ \\
\hline Carbohydrates ( $\%$ of energy) & 49.52 & 7.73 & 48.03 & 7.46 & 49.06 & 7.76 & $50 \cdot 00$ & 7.52 & $<0.001$ \\
\hline Dietary fibre $(\mathrm{g} / \mathrm{d})$ & 19.55 & 5.33 & $21 \cdot 80$ & $5 \cdot 12$ & $23 \cdot 40$ & $5 \cdot 17$ & $25 \cdot 54$ & $5 \cdot 10$ & $<0.001$ \\
\hline$n-3$ Fatty acids $(g / d)$ & 1.56 & 0.53 & 1.67 & 0.53 & 1.73 & 0.57 & $2 \cdot 00$ & 0.53 & $<0.001$ \\
\hline Vitamin $B_{1}(\mathrm{mg} / \mathrm{d})$ & $2 \cdot 06$ & 0.53 & 1.80 & 0.53 & $1 \cdot 78$ & 0.28 & $1 \cdot 75$ & 0.53 & $<0.001$ \\
\hline Vitamin $B_{6}(\mathrm{mg} / \mathrm{d})$ & 1.80 & 0.26 & 1.97 & 0.26 & $2 \cdot 04$ & 0.28 & 2.09 & 0.26 & $<0.001$ \\
\hline $\mathrm{Fe}(\mathrm{mg} / \mathrm{d})$ & 18.93 & 2.93 & $17 \cdot 62$ & 2.96 & $17 \cdot 34$ & $3 \cdot 16$ & $16 \cdot 62$ & 2.95 & $<0.001$ \\
\hline \multicolumn{10}{|l|}{ Food groups } \\
\hline Red meat $(\mathrm{g} / \mathrm{d})$ & 85.89 & 39.73 & $86 \cdot 06$ & $39 \cdot 12$ & $78 \cdot 81$ & 39.39 & 63.45 & 39.49 & $<0.001$ \\
\hline Whole grains $(\mathrm{g} / \mathrm{d})$ & 21.76 & 74.92 & 39.48 & 73.92 & $46 \cdot 52$ & $74 \cdot 19$ & 61.59 & 74.43 & $<0.001$ \\
\hline Fruit $(\mathrm{g} / \mathrm{d})$ & 162.01 & 191.45 & 234.49 & 188.60 & $301 \cdot 12$ & $189 \cdot 80$ & $417 \cdot 18$ & 189.97 & $<0.001$ \\
\hline Vegetables $(\mathrm{g} / \mathrm{d})$ & 176.57 & $110 \cdot 65$ & $222 \cdot 81$ & $109 \cdot 00$ & 261.05 & $109 \cdot 85$ & $292 \cdot 62$ & $109 \cdot 89$ & $<0.001$ \\
\hline Nuts, soya and legumes (g/d) & $43 \cdot 72$ & 35.46 & 57.77 & 35.07 & 61.97 & $35 \cdot 37$ & 64.58 & 35.46 & $<0.001$ \\
\hline Low-fat dairy products $(\mathrm{g} / \mathrm{d})$ & 301.67 & 259.71 & 312.03 & $256 \cdot 32$ & 344.58 & 257.95 & $376 \cdot 15$ & 258.22 & $<0.001$ \\
\hline High-fat dairy products $(\mathrm{g} / \mathrm{d})$ & $15 \cdot 24$ & $17 \cdot 33$ & $15 \cdot 37$ & 16.99 & 14.49 & $17 \cdot 25$ & $13 \cdot 74$ & $17 \cdot 19$ & 0.25 \\
\hline Refined grains $(\mathrm{g} / \mathrm{d})$ & $480 \cdot 77$ & $155 \cdot 72$ & 401.55 & 153.52 & 375.69 & $154 \cdot 71$ & 317.08 & $154 \cdot 77$ & $<0.001$ \\
\hline Trans-fatty acids (g/d) & 0.29 & $0 \cdot 10$ & 0.24 & 0.10 & 0.19 & $0 \cdot 11$ & 0.14 & 0.10 & $<0.001$ \\
\hline Sugar-sweetened beverages and fruit juice (g/d) & $66 \cdot 67$ & 72.52 & $60 \cdot 19$ & 71.50 & $52 \cdot 37$ & 71.89 & 39.56 & $72 \cdot 01$ & $<0.001$ \\
\hline Hydrogenated vegetable oils $(\mathrm{g} / \mathrm{d})$ & 9.88 & $10 \cdot 66$ & 11.38 & 10.52 & $10 \cdot 21$ & $10 \cdot 64$ & $10 \cdot 07$ & 10.47 & 0.03 \\
\hline
\end{tabular}

* Energy intake is adjusted for age and sex; all other values are adjusted for age, sex and energy intake.

† Obtained from ANCOVA.

$95 \%$ CI $0.44,0.78)$. After controlling for potential confounders, this association was strengthened (OR 0.51; $95 \%$ CI $0.35,0.72$ ). Participants with the highest adherence to AHEI-2010 had $36 \%$ lower odds of depression compared with those with the lowest adherence (OR 0.64; 95\% CI 0.51, 0.79). This association remained significant even after taking potential confounders into account (OR 0.55; 95\% CI 0.42, 0.72). In addition, a decreasing trend in the odds of anxiety and depression was seen with increasing quartiles of AHEI-2010 ( $P_{\text {trend }}<0.001$ for all models). 

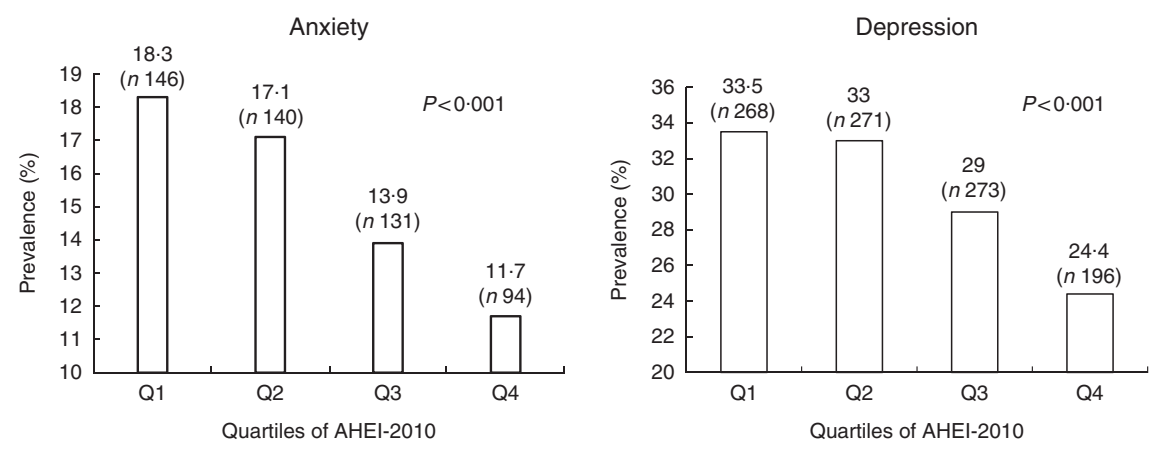

Fig. 1. The prevalence of anxiety and depression in study participants across quartiles of Alternative Healthy Eating Index-2010 (AHEI-2010).

Among men, those in the highest quartile of AHEI-2010 were 45 and $37 \%$ less likely to have anxiety and depression, compared with those in the lowest category (OR 0.55; $95 \%$ CI 0.32, 0.94 for anxiety and OR 0.63; 95\% CI 0.43, 0.91 for depression). However, after controlling for potential cofounders, there were no significant associations between adherence to AEHI-2010 and frequency of anxiety or depression in men (OR $0.51 ; 95 \%$ CI $0.25,1.04$ for anxiety and OR 0.70; $95 \%$ CI 0.44, 1.11 for depression). Women in the highest categories of AHEI-2010 had 49 and $45 \%$ lower odds of having anxiety and depression (OR $0.51 ; 95 \%$ CI $0 \cdot 36,0.72$ for anxiety and OR 0.55; $95 \% \mathrm{CI}$ $0.42,0.73$ for depression), compared with those in the lowest category. Adjustment for potential cofounders did not change these associations.

Stratified analysis by age revealed that among individuals who were 40 years old or younger, after adjustment for different confounders, those in the top category were at a 58 and $51 \%$ lower odds for having anxiety and depression than those in the bottom category (OR 0.42; $95 \%$ CI 0.27, 0.65 for anxiety and OR $0.49 ; 95 \%$ CI 0.35 , 0.68 for depression). However, after accounting for known potential confounders, no significant associations were found between adherence to AHEI-2010 and mental disorders in those $>40$ years old (OR 0.72; 95\% CI 0.41, 1.25 for anxiety and OR 0.76; $95 \%$ CI 0.50, 1.17 for depression).

\section{Discussion}

We found a significant inverse association between adherence to AHEI-2010 and odds of anxiety and depression among Iranian adults. This association remained significant even after adjustments for potential confounders, including energy intake and BMI. In stratified analysis, we found protective associations between adherence to AHEI-2010 and mental disorders in women, as well as in individuals who were 40 years old or younger.

Mental health disorders are globally increasing conditions associated with poor quality of life and social outcomes ${ }^{(4,5,22)}$. In addition, these illnesses impose a great burden to the healthcare system ${ }^{(22)}$. Therefore, improvement in dietary intake might help prevent these conditions ${ }^{(8)}$. Some studies have examined the associations between dominant dietary patterns and mental disorders by means of a posteriori approaches ${ }^{(6,23-26)}$. A recent case-control study from Iran has shown that adherence to healthy dietary pattern, identified by factor analysis, was associated with a decreased risk of major depression. However, no significant association was seen between unhealthy dietary pattern and depression ${ }^{(26)}$. Jacka et al. ${ }^{(24)}$ have also shown that adherence to traditional dietary pattern was associated with a lower risk of depression and anxiety in Australian women. This association was attributed to the high content of vegetables, fruits, meat, fish and whole grains in this pattern. In addition, a positive association was observed between Western dietary pattern (characterised by processed or fried foods, refined grains, sugary products and beer) and mental disorders, in this study ${ }^{(24)}$. Similar findings were reported from prospective cohort studies ${ }^{(6,25)}$. However, the investigators from Nurses' Health Study did not reach such a clear association between consumption of prudent and Western dietary patterns and depression ${ }^{(23)}$. Moreover, some other studies failed to find any association between dietary patterns identified by a posteriori methods and depression in other communities ${ }^{(27,28)}$. Although a posteriori-driven dietary patterns have been greatly used to assess diet-disease relations, it seems that using a priori methods for the identification of dietary patterns can provide more useful information about the adherence of general population to the dietary guidelines ${ }^{(8-10)}$. Earlier studies on dietary patterns derived from a priori methods have mostly been confined to developed countries $^{(9-11)}$. Findings from the Whitehall II study revealed that women with high scores of AHEI had a lower risk of recurrent depressive symptoms ${ }^{(9)}$. Moreover, Kuczmarski et $a l .{ }^{(10)}$ have indicated that high diet quality, identified by HEI-2005, was inversely associated with depressive symptoms. Similar findings were also reported from other studies ${ }^{(11)}$. Greater adherence to the Mediterranean diet (Med-Diet) was also protectively linked with depression in a large cohort study of Spanish adults ${ }^{(29)}$. Our findings are in accordance with the above-mentioned studies, in which greater adherence to AHEI was related to the lower odds of depression.

We also found a significant protective association between adherence to AHEI-2010 and anxiety. Although anxiety is highly prevalent worldwide ${ }^{(1)}$, scarce data are available regarding the association between AHEI and anxiety. A cross-sectional study among Iranian adults showed that high adherence to traditional, Western and fast food dietary patterns was associated with an increased risk of psychological disorders including anxiety. However, a lacto-vegetarian dietary pattern that contained high 
Table 3. Anxiety and depression across quartiles of Alternative Healthy Eating Index-2010 (AHEI-2010) in the whole population, as well as stratified by sex and age group*

(Multivariable-adjusted odds ratios and $95 \%$ confidence intervals)

\begin{tabular}{|c|c|c|c|c|c|c|c|c|}
\hline & \multicolumn{7}{|c|}{ Quartiles of AHEI-2010 } & \multirow[b]{3}{*}{$P_{\text {trend }} t$} \\
\hline & \multirow{2}{*}{$\frac{\mathrm{Q}_{1}}{\mathrm{OR}}$} & \multicolumn{2}{|c|}{$\mathrm{Q}_{2}$} & \multicolumn{2}{|c|}{$\mathrm{Q}_{3}$} & \multicolumn{2}{|c|}{$\mathrm{Q}_{4}$} & \\
\hline & & OR & $95 \% \mathrm{Cl}$ & OR & $95 \% \mathrm{Cl}$ & OR & $95 \% \mathrm{Cl}$ & \\
\hline \multicolumn{9}{|c|}{ Whole population ( $n$ 3363) } \\
\hline \multicolumn{9}{|c|}{ Anxiety } \\
\hline Crude & 1.00 & 0.92 & $0.71,1.19$ & 0.72 & $0.56,0.93$ & 0.59 & $0.44,0.78$ & $<0.001$ \\
\hline Model 1 & 1.00 & 0.83 & $0.63,1.11$ & 0.65 & $0.48,0.86$ & 0.47 & $0.34,0.64$ & $<0.001$ \\
\hline Model 2 & 1.00 & 0.94 & $0.69,1.28$ & 0.65 & $0.47,0.90$ & 0.51 & $0.35,0.72$ & $<0.001$ \\
\hline \multicolumn{9}{|l|}{ Depression } \\
\hline Crude & 1.00 & 0.98 & $0.79,1 \cdot 20$ & 0.81 & $0.66,0.99$ & 0.64 & $0.51,0.79$ & $<0.001$ \\
\hline Model 1 & 1.00 & 0.90 & $0.71,1.13$ & 0.68 & $0.54,0.86$ & 0.50 & $0.39,0.64$ & $<0.001$ \\
\hline Model 2 & 1.00 & 0.91 & $0.71,1 \cdot 17$ & 0.65 & $0.50,0.84$ & 0.55 & $0.42,0.72$ & $<0.001$ \\
\hline \multicolumn{9}{|l|}{ Men ( $n$ 1403) } \\
\hline \multicolumn{9}{|l|}{ Anxiety } \\
\hline Crude & 1.00 & 0.90 & $0.58,1.38$ & 0.71 & $0.45,1 \cdot 11$ & 0.55 & $0.32,0.94$ & 0.02 \\
\hline Model 1 & 1.00 & 0.94 & $0.56,1.56$ & 0.91 & $0.54,1.53$ & 0.53 & $0.27,1.01$ & 0.09 \\
\hline \multirow{2}{*}{\multicolumn{9}{|c|}{ Depression }} \\
\hline & & & & & & & & \\
\hline Crude & 1.00 & 0.79 & $0.56,1.09$ & 0.72 & $0.51,1.01$ & 0.63 & $0.43,0.91$ & 0.01 \\
\hline Model 1 & 1.00 & 0.81 & $0.56,1.19$ & 0.72 & $0.49,1.06$ & 0.61 & $0.40,0.94$ & 0.02 \\
\hline Model 2 & 1.00 & 0.84 & $0.56,1.27$ & 0.72 & $0.48,1.10$ & 0.70 & $0.44,1.11$ & 0.08 \\
\hline \multicolumn{9}{|c|}{ Women ( $n$ 1960) } \\
\hline \multicolumn{9}{|l|}{ Anxiety } \\
\hline Crude & 1.00 & 0.86 & $0.62,1 \cdot 19$ & 0.64 & $0.46,0.88$ & 0.51 & $0.36,0.72$ & $<0.001$ \\
\hline Model 1 & 1.00 & 0.79 & $0.56,1.21$ & 0.56 & $0.40,0.79$ & 0.44 & $0.31,0.64$ & $<0.001$ \\
\hline Model 2 & 1.00 & 1.01 & $0.68,1.49$ & 0.58 & $0.39,0.87$ & 0.51 & $0.33,0.78$ & $<0.001$ \\
\hline \multicolumn{9}{|l|}{ Depression } \\
\hline Crude & 1.00 & 1.04 & $0.79,1.37$ & 0.76 & $0.58,0.99$ & 0.55 & $0.42,0.73$ & $<0.001$ \\
\hline Model 1 & 1.00 & 0.95 & $0.71,1.27$ & 0.66 & $0.50,0.88$ & 0.47 & $0.35,0.64$ & $<0.001$ \\
\hline Model 2 & 1.00 & 0.98 & $0.71,1.37$ & 0.61 & $0.44,0.85$ & 0.51 & $0.36,0.71$ & $<0.001$ \\
\hline \multicolumn{9}{|c|}{ Age $\leq 40$ years $(n 2125)$} \\
\hline \multicolumn{9}{|l|}{ Anxiety } \\
\hline Crude & 1.00 & 0.96 & $0.70,1.31$ & 0.81 & $0.59,1.11$ & 0.51 & $0.35,0.73$ & $<0.001$ \\
\hline Model 1 & 1.00 & 0.90 & $0.65,1 \cdot 24$ & 0.71 & $0.51,0.98$ & 0.43 & $0.29,0.63$ & $<0.001$ \\
\hline Model 2 & 1.00 & 0.96 & $0.68,1.38$ & 0.68 & $0.47,0.99$ & 0.42 & $0.27,0.65$ & $<0.001$ \\
\hline \multicolumn{9}{|l|}{ Depression } \\
\hline Crude & 1.00 & 1.04 & $0.81,1.34$ & 0.80 & $0.62,1.03$ & 0.56 & $0.42,0.75$ & $<0.001$ \\
\hline Model 1 & 1.00 & 0.96 & $0.74,1.25$ & 0.68 & $0.52,0.89$ & 0.46 & $0.34,0.62$ & $<0.001$ \\
\hline Model 2 & 1.00 & 0.95 & $0.71,1.27$ & 0.62 & $0.46,0.84$ & 0.49 & $0.35,0.68$ & $<0.001$ \\
\hline \multicolumn{9}{|c|}{ Age $>40$ years $(n 1238)$} \\
\hline \multicolumn{9}{|l|}{ Anxiety } \\
\hline Crude & 1.00 & 0.84 & $0.53,1.33$ & 0.58 & $0.36,0.92$ & 0.73 & $0.46,1.15$ & 0.08 \\
\hline Model 1 & 1.00 & 0.73 & $0.45,1.18$ & 0.47 & $0.29,0.77$ & 0.60 & $0.37,0.96$ & 0.02 \\
\hline Model 2 & 1.00 & 0.83 & $0.47,1.46$ & 0.48 & $0.27,0.86$ & 0.72 & $0.41,1.25$ & 0.11 \\
\hline \multicolumn{9}{|l|}{ Depression } \\
\hline Crude & 1.00 & 0.86 & $0.60,1.24$ & 0.82 & $0.58,1 \cdot 16$ & 0.75 & $0.53,1.07$ & 0.12 \\
\hline Model 1 & 1.00 & 0.83 & $0.56,1.22$ & 0.75 & $0.52,1.08$ & 0.65 & $0.45,0.94$ & 0.02 \\
\hline Model 2 & 1.00 & 0.88 & $0.56,1.36$ & 0.75 & $0.50,1 \cdot 15$ & 0.76 & $0.50,1.17$ & 0.17 \\
\hline
\end{tabular}

* Model 1: adjusted for age, sex, energy intake and BMI. Model 2: further adjustment for physical activity, smoking, marital status, education, family size, house possession, diabetes, intake of anti-psychotic medications and dietary supplements. In stratified analysis by sex: model 1: adjusted for age, energy intake and BMI. Model 2: further adjustment for physical activity, smoking, marital status, education, family size, house possession, diabetes, intake of anti-psychotic medications and dietary supplements. In stratified analysis by age: model 1: adjusted for sex, energy intake and BMI. Model 2: further adjustment for physical activity, smoking, marital status, education, family size, house possession, diabetes, intake of anti-psychotic medications and dietary supplements.

t Obtained by the use of categories of $\mathrm{AHEl}-2010$ as an ordinal variable in the model.

intakes of fruits, vegetables and low-fat dairy products was protectively associated with anxiety ${ }^{(30)}$. Similar findings were reported from an Australian cohort study, in which Western dietary pattern was related to an increased risk of anxiety $^{(31)}$. Antonogeorgos et al. ${ }^{(32)}$ have also indicated that low adherence to the Med-Diet might mediate the unfavourable effect of anxiety on CVD risk factors in the Greek population. Moreover, in a cross-sectional study, greater consumption of processed foods was associated with an increased risk of anxiety in the Iranian population ${ }^{(33)}$. It is worth mentioning that using different methods to assess mental health might explain these inconsistent results.

We found that adherence to AHEI-2010 was related to mental disorders in women, but a non-significant association was seen in men. Lower prevalence of anxiety and depression in male participants compared with female participants might explain 
these non-significant associations (10.8 v. 18.3\% for anxiety; 22.9 v. $35.1 \%$ for depression).

In the present study, we used OR from logistic regression models; these estimates in cross-sectional studies may not be valid estimators of the rate ratios when the binary outcome variable has a high prevalence $(>10 \%)^{(34)}$ and will overestimate the risk ratio when it is more than 1 or underestimate the risk ratio when it is less than 1 . Some formulas have been suggested to correct the adjusted OR obtained from logistic regression to derive an estimate of association that better represents the true relative risk ${ }^{(35)}$. Although the prevalence of anxiety and depression in our study was 15.2 and $30.0 \%$, respectively, we did not make correction, because if the OR was $>2.5$ or $<0.5$ correction of the OR might be desirable to more appropriately interpret the magnitude of an association. It is worth noting that using these corrections may attenuate the obtained OR.

Several plausible mechanisms might explain the inverse association of AHEI-2010 with depression. This association has been generated by the cumulative effects of all components of AHEI-2010 rather than an individual nutrient or food group ${ }^{(9,10)}$. High content of folate, $\mathrm{B}$ vitamins and antioxidants in the healthy eating pattern might reduce neuronal damage of oxidative stress $^{(4,13,36,37)}$. Because of the relationship between high levels of inflammatory biomarkers and depressive symptoms, anti-inflammatory properties of foods included in AHEI have been shown to reduce concentrations of monoamines $^{(9,10,13)}$. Moreover, high levels of PUFA and $n-3$ fatty acids presented in oily fish and other components of AHEI are other possible mechanisms ${ }^{(8,9)}$.

The strength of this study is the large included population, whereas previous studies have mostly been performed on small sample sizes. The associations that we identified are independent of many factors, because of adjustments for several potential confounders. However, several limitations should be considered while interpreting our findings. The major limitation is the cross-sectional design of the study, which does not allow inferring causality. Large prospective cohort studies are required to provide an evidence for the causal relationship. In addition, using FFQ to assess usual dietary intake is subjected to many errors depending on memory, fixed list of foods and portion sizes. However, we use a validated FFQ for assessment of dietary intakes ${ }^{(14)}$. Furthermore, the study might be susceptible to recall bias because of low response rate and misclassification. The study population consisted of a medical university nonacademic staff, including crews, employees and managers. We excluded some university teaching hospitals and research centres to reduce the conflict of interest in research. Although the socio-economic status of the study population was representative of the general Iranian population, extrapolating the findings to other populations might be done cautiously. Lack of having a non-representative population can be harmful or beneficial depending on the study question and context. The important point is that the distortions may be in any direction (which is unpredictable). In that respect, our findings may be subject to selection bias. We assessed mental health by means of a self-administered questionnaire, which might lead to misclassification of study participants. It is worth noting that the validity of the HADS questionnaire has been previously examined among the Iranian population ${ }^{(20)}$. Moreover, despite controlling for a wide range of potential confounders, the effects of residual confounders cannot be excluded.

In conclusion, we found evidence indicating that greater adherence to AHEI-2010 was associated with a lower odds of anxiety and depression. More adherence to AHEI-2010 was associated with a reduced risk of mental disorders in women, as well as in those who were 40 years old or younger. These initial findings need to be confirmed with prospective assessments in order to clarify the true casual association between AHEI-2010 and mental disorders.

\section{Acknowledgements}

This study was extracted from a PhD dissertation that was approved by School of Nutrition and Food Sciences, Isfahan University of Medical Sciences (no. 394292). The authors wish to thank all staff of Isfahan University of Medical Sciences who kindly participated in the study and staff of Public Relations Unit, and other authorities of IUMS for their excellent cooperation.

The financial support for this study comes from Food Security Research Center, Isfahan University of Medical Sciences, Isfahan, Iran. Food Security Research Center has no role in conception, design, data analysis and manuscript drafting. P. S., M. H., A. H. K., H. A., A. E. and P. A. contributed to conception, design, data collection, statistical analyses, data interpretation, manuscript drafting, approval of the final version of the manuscript and agreed for all aspects of the work.

None of the authors had any personal or financial conflicts of interest.

\section{References}

1. Baxter AJ, Scott KM, Vos T, et al. (2013) Global prevalence of anxiety disorders: a systematic review and meta-regression. Psychol Med 43, 897-910.

2. Steel Z, Marnane C, Iranpour C, et al. (2014) The global prevalence of common mental disorders: a systematic review and meta-analysis 1980-2013. Int J Epidemiol 43, 476-493.

3. Noorbala AA, Bagheri Yazdi SA, Yasamy MT, et al. (2004) Mental health survey of the adult population in Iran. Br J Psychiatry 184, 70-73.

4. Popa TA \& Ladea M (2012) Nutrition and depression at the forefront of progress. J Med Life 5, 414-419.

5. Quirk SE, Williams LJ, O'Neil A, et al. (2013) The association between diet quality, dietary patterns and depression in adults: a systematic review. BMC Psychiatry 13, 175.

6. Chan R, Chan D \& Woo J (2014) A prospective cohort study to examine the association between dietary patterns and depressive symptoms in older Chinese people in Hong Kong. PLOS ONE 9, e105760.

7. Khosravi M, Sotoudeh G, Raisi F, et al. (2014) Comparing dietary patterns of depressed patients versus healthy people in a case control protocol. BMJ Open 4, e003843.

8. Lai JS, Hiles S, Bisquera A, et al. (2014) A systematic review and meta-analysis of dietary patterns and depression in community-dwelling adults. Am J Clin Nutr 99, 181-197.

9. Akbaraly TN, Sabia S, Shipley MJ, et al. (2013) Adherence to healthy dietary guidelines and future depressive symptoms: 
evidence for sex differentials in the Whitehall II study. Am J Clin Nutr 97, 419-427.

10. Kuczmarski MF, Cremer Sees A, Hotchkiss L, et al. (2010) Higher Healthy Eating Index-2005 scores associated with reduced symptoms of depression in an urban population: findings from the Healthy Aging in Neighborhoods of Diversity Across the Life Span (HANDLS) study. I Am Diet Assoc 110, 383-389.

11. Loprinzi PD \& Mahoney S (2014) Concurrent occurrence of multiple positive lifestyle behaviors and depression among adults in the United States. J Affect Disord 165, 126-130.

12. Beydoun MA \& Wang Y (2010) Pathways linking socioeconomic status to obesity through depression and lifestyle factors among young US adults. I Affect Disord $\mathbf{1 2 3}$, 52-63.

13. Assies J, Pouwer F, Lok A, et al. (2010) Plasma and erythrocyte fatty acid patterns in patients with recurrent depression: a matched case-control study. PLOS ONE 5, e10635.

14. Keshteli A, Esmaillzadeh A, Rajaie S, et al. (2014) A dish-based semi-quantitative food frequency questionnaire for assessment of dietary intakes in epidemiologic studies in Iran: design and development. Int J Prev Med 5, 29-36.

15. Barak F, Falahi E, Keshteli AH, et al. (2015) Red meat intake, insulin resistance, and markers of endothelial function among Iranian women. Mol Nutr Food Res 59, 315-322.

16. Saneei P, Fallahi E, Barak F, et al. (2015) Adherence to the DASH diet and prevalence of the metabolic syndrome among Iranian women. Eur J Nutr 54, 421-428.

17. Salehi-Abargouei A, Esmaillzadeh A, Azadbakht L, et al. (2016) Nutrient patterns and their relation to general and abdominal obesity in Iranian adults: findings from the SEPAHAN study. Eur J Nutr 55, 505-518.

18. Chiuve SE, Fung TT, Rimm EB, et al. (2012) Alternative dietary indices both strongly predict risk of chronic disease. J Nutr 142, 1009-1018.

19. Willett WC (1998) Nutritional Epidemiology, 2nd ed. New York: Oxford University Press.

20. Montazeri A, Vahdaninia M, Ebrahimi M, et al. (2003) The Hospital Anxiety and Depression Scale (HADS): translation and validation study of the Iranian version. Health Qual Life Outcomes 1, 14.

21. National Institute for Health and Clinical Excellence (2006) Promoting and Creating Built or Natural Environments That Encourage and Support Physcial Activity: Scope. NICE: London.

22. Kessler RC \& Bromet EJ (2013) The epidemiology of depression across cultures. Annu Rev Public Health 34, 119-138.

23. Chocano-Bedoya PO, O'Reilly EJ, Lucas M, et al. (2013) Prospective study on long-term dietary patterns and incident depression in middle-aged and older women. Am J Clin Nutr 98, 813-820.

24. Jacka FN, Cherbuin N, Anstey KJ, et al. (2014) Dietary patterns and depressive symptoms over time: examining the relationships with socioeconomic position, health behaviours and cardiovascular risk. PLOS ONE 9, e87657.

25. Le Port A, Gueguen A, Kesse-Guyot E, et al. (2012) Association between dietary patterns and depressive symptoms over time: a 10-year follow-up study of the GAZEL cohort. PLOS ONE 7, e51593.

26. Rashidkhani B, Pourghassem Gargari B, Ranjbar F, et al. (2013) Dietary patterns and anthropometric indices among Iranian women with major depressive disorder. Psychiatry Res 210, 115-120.

27. Gougeon L, Payette H, Morais J, et al. (2015) Dietary patterns and incidence of depression in a cohort of communitydwelling older canadians. J Nutr Health Aging 19, 431-436.

28. Sugawara N, Yasui-Furukori N, Tsuchimine S, et al. (2012) No association between dietary patterns and depressive symptoms among a community-dwelling population in Japan. Ann Gen Psychiatry 11, 24.

29. Sanchez-Villegas A, Martinez-Gonzalez MA, Estruch R, et al. (2013) Mediterranean dietary pattern and depression: the PREDIMED randomized trial. BMC Med 11, 208.

30. Hosseinzadeh M, Vafa M, Esmaillzadeh A, et al. (2016) Empirically derived dietary patterns in relation to psychological disorders. Public Health Nutr 19, 204-217.

31. Jacka FN, Pasco JA, Mykletun A, et al. (2010) Association of Western and traditional diets with depression and anxiety in women. Am J Psychiatry 167, 305-311.

32. Antonogeorgos G, Panagiotakos DB, Pitsavos C, et al. (2012) Understanding the role of depression and anxiety on cardiovascular disease risk, using structural equation modeling; the mediating effect of the Mediterranean diet and physical activity: the ATTICA study. Ann Epidemiol 22, 630-637.

33. Bakhtiyari M, Ehrampoush E, Enayati N, et al. (2013) Anxiety as a consequence of modern dietary pattern in adults in Tehran - Iran. Eat Behav 14, 107-112.

34. Greenland S (1987) Interpretation and choice of effect measures in epidemiologic surveys. Am J Epidemiol 125, 761-768.

35. Zhang J \& Yu KF (1998) What's the relative risk? A method of correcting the odds ratio in cohort studies of common outcomes. JAMA 280, 1690-1691.

36. Ibarra O, Gili M, Roca M, et al. (2014) The Mediterranean diet and micronutrient levels in depressive patients. Nutr Hosp $\mathbf{3 1}$, $1171-1175$

37. Gougeon L (2014) Nutritional predictors of depression in a cohort of community-dwelling elderly Canadians: NuAge cohort. Appl Physiol Nutr Metab 39, 1412. 